\title{
Nota sobre morcegos (Mammalia, Chiroptera) e moscas ectoparasitas (Insecta, Diptera) do Parque Nacional da Serra do Pardo, estado do Pará, Brasil Note on bats (Mammalia, Chiroptera) and ectoparasite flies (Insecta, Diptera) from Serra do Pardo National Park, Pará state, Brazil
}

\author{
Jennifer Bandeira Silva' (1) Liriann Chrisley Nascimento Da Silval (1) | Karina Dias-Silva' (1) \\ Angelino Pereira de Oliveira Júnior (1) | Brenda Tayná Sousa da Silvall (D) | Geysa Kelly Oliveira Veloso' (1) \\ Karen Monteiro Moy' (1) | Paula da Conceição Praxedes Santana ${ }^{\text {III }}$ (1) | Rozinete Francisca Rezende' (1) \\ Thais Sousa Martins ${ }^{\text {II }}$ (I) Thiago Bernardi Vieiral \\ 'Universidade Federal do Pará. Altamira, Pará, Brasil \\ "Universidade Estadual de Feira de Santana. Feira de Santana, Bahia, Brasil \\ "'Universidade Federal do Pará. Bragança, Pará, Brasil
}

\begin{abstract}
Resumo: Os morcegos apresentam grande diversidade de hábitos alimentares, o que faz com que possam ser utilizados como indicadores de qualidade ambiental, além de prestarem serviços ecossistêmicos, como dispersão de sementes, estruturação de comunidade de plantas e controle de insetos. Além disso, o estudo da interação de morcegos com ectoparasitas pode possibilitar melhor compreensão dos processos ecológicos e evolutivos associados. Dessa forma, o objetivo desse estudo é contribuir para o conhecimento de algumas espécies de morcegos e suas moscas ectoparasitas no Parque Nacional da Serra do Pardo, interflúvio Xingu-Tapajós, estado do Pará, Brasil. A captura dos morcegos foi realizada em quatro pontos amostrais, através de redes de neblina e busca ativa. Em cada indivíduo capturado foi realizada inspeção da pelagem e das asas para retirada das moscas ectoparasitas. Foram amostrados 13 indivíduos de morcegos, representados por duas famílias (Phyllostomidae e Molossidae), seis gêneros e seis táxons, sendo C. perspicillata a mais amostrada. Quanto à guilda trófica, observamos espécies insetívoras e frugívoras, sendo a última a mais abundante. Para moscas ectoparasitas, observamos apenas a família Streblidae. Todos os ectoparasitas estavam relacionados aos indivíduos de C. perspicillata.
\end{abstract}

Palavras-chave: Área de proteção. Conservação. Espécies indicadoras de qualidade. Lista de espécies. Terra do Meio.

\begin{abstract}
Bats have a great diversity of eating habits, means that they can be used as indicators of environmental quality, in addition to providing ecosystem services such as: seed dispersal, structuring of plant communities and insect control. In addition, the interaction of bats with ectoparasites may allow a better understanding of the associated ecological and evolutionary processes. The main of this work is contribute for the knowledge of bats and their ectoparasite fly species from Serra do Pardo National Park, Xingu-Tapajos interfluve, Pará state, Brazil. The capture of the bats was performed in four sampling points, through fog networks and active search. Each bat individual captured, the coat and wings were inspected to remove ectoparasitic flies. We sampled 13 bat individuals, represented by two families (Phyllostomidae and Molossidae), six genera and six species, being C. perspicillata the most abundant. As for the trophic guild, we observed insectivorous and frugivorous species, being the latter the most abundant species. For ectoparasite flies, we observed only the family Streblidae. All ectoparasites were related to C. perspicillata individuals.
\end{abstract}

Keywords: Protected areas. Conservation. Quality indicator species. Species list. Terra do Meio.

SILVA, J. B., L. C. N. DA SILVA, K. DIAS-SILVA, A. P. OLIVEIRA JÚNIOR, B. T. S. SILVA, G. K. O. VELOSO, K. M. MOY, P. C. P. SANTANA, R. F. REZENDE, T. S. MARTINS \& T. B. VIEIRA, 2020. Nota sobre morcegos (Mammalia, Chiroptera) e moscas ectoparasitas (Insecta, Diptera) do Parque Nacional da Serra do Pardo, estado do Pará, Brasil. Boletim do Museu Paraense Emílio Goeldi. Ciências Naturais 15(3): 829-841. DOI: http://doi.org/10.46357/bcnaturais.v15i3.263.

Autora para correspondência: Liriann Chrisley Nascimento da Silva. Universidade Federal do Pará. Campus Altamira. Programa de PósGraduação em Biodiversidade e Conservação. Rua Coronel José Porfírio, 2515 - São Sebastião. Altamira, PA, Brasil. CEP 68372-040 (lirianncns@gmail.com).

Recebido em 22/01/2020

Aprovado em 22/11/2020

Responsabilidade editorial: Alexandra Maria Ramos Bezerra
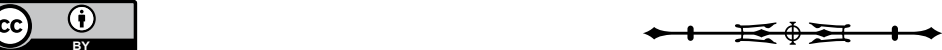


\section{INTRODUÇÃO}

A região da Terra do Meio é uma das áreas contínuas conservadas de alta importância nacional e mundial (ICMBio, 2012; ARPA, 2015). O termo 'Terra do Meio' foi criado na década de 1970, durante a abertura das rodovias Transamazônica (BR-230) e Cuiabá-Santarém (BR-163), que, associadas com a abertura da estrada da Canopus (Transiriri), ligando o rio Iriri a São Félix do Xingu, na década seguinte, propagaram uma série de atividades conflitantes (Escada et al., 2005; ARPA, 2015). A região está situada às margens do rio Xingu, localizada entre os municípios de Altamira e São Félix do Xingu, abrangendo cerca de 8,5 milhões de hectares, ocupando cerca de 50\% do território paraense (ARPA, 2015). A Terra do Meio é composta por 11 áreas de proteção (AP), formando um mosaico de unidades de conservação (UC) de proteção integral e uso sustentável e territórios indígenas (TI). Entre essas UC, encontra-se o Parque Nacional da Serra do Pardo (PNSP), estrategicamente delimitado para garantir a conservação dos recursos naturais e minerais (Velásquez et al., 2006; ARPA, 2015). Criado sob o Decreto s/n de 17 de fevereiro de 2005 (Brasil, 2005), o PNSP é uma UC federal de proteção integral amazônica, com 446.552 ha (Barros, 2010; ARPA, 2015). Apesar do histórico de uso por colonos, comunidades tradicionais e fazendeiros, o parque ainda mantém cerca de 95\% da sua vegetação nativa (Barros, 2010; Fávaro, 2011). Essa área de extrema importância para conservação vem sendo ameaçada por atividades de mineração, obras de infraestrutura planejadas e, principalmente, pela pecuária (Velásquez et al., 2006; Aleixo, 2009; Develey, 2009). Em relação à fauna e à flora do PNSP, alguns estudos foram realizados para o desenvolvimento do Plano de Manejo (ARPA, 2015), porém informações sobre a quiropterofauna não foram contempladas no Plano de Manejo ou em estudos pretéritos.

Aordem Chiroptera é a segunda mais especiosa entre os mamíferos (Paglia et al., 2012; Nogueira et al. , 2014).
Atualmente, são reconhecidas aproximadamente 1.400 espécies de morcegos no mundo (Simmons \& Cirranello, 2020), das quais 181 ocorrem no Brasil (Garbino et al., 2020). Os morcegos apresentam grande diversidade de hábitos alimentares, com animais insetívoros, nectarívoros, piscívoros, frugívoros, onívoros, carnívoros ou hematófagos (Kunz et al., 2011). Os efeitos na estruturação de comunidades de plantas (Fleming \& Heithaus, 1981; Kunz et al., 2011) e a atuação no controle de populações de insetos (Williams-Guillén et al., 2008; Kalka et al., 2008), inclusive de pragas agrícolas (Cleveland et al., 2006; Ghanem \& Voigt, 2012), reforçam o reconhecimento desse grupo como espécieschave em florestas tropicais. Os morcegos também têm importante relação com os ectoparasitas, em especial os dípteros das famílias Streblidae e Nycteribiidae (Rui \& Graciolli, 2005; Graciolli \& Bianconi, 2007; Santos, F. et al., 2012). Essas duas famílias estão distribuídas por toda a região Neotropical (Frank et al. , 2014), compondo cerca de 96 espécies de Streblidae (Graciolli, 2020) e 26 espécies de Nycteribiidae (Graciolli et al., 2007) para o continente americano. Geralmente, o parasitismo é em espécie específica e a infestação pode ser relacionada às características intrínsecas do hospedeiro, como idade, sexo, estado reprodutivo e de saúde (Rui \& Graciolli, 2005; Santos, F. et al., 2012). Porém, os estudos envolvendo parasitismo são principalmente voltados à taxonomia, às taxas de infestação, à prevalência e às variações morfológicas, enquanto que a relação parasitohospedeiro de dípteros em morcegos vem sendo explorada apenas em estudos mais recentes (Prevedello et al., 2005; Rui \& Graciolli, 2005; Silva \& Ortêncio Filho, 2011; Santos, F. et al., 2012).

Lacunas de conhecimento dificultam estratégias eficientes de conservação em unidades de conservação (Hopkins, 2007; Collen et al., 2008; Sousa-Baena et al., 2014; Oliveira et al., 2016) e esse déficit ainda é mais comum nas regiões tropicais (Kier et al., 2005; Collen et al., 2008; Xu et al., 2017), inclusive a Amazônia, que abriga 
unidades em 49\% de sua extensão (Oliveira et al., 2017). O planejamento de estratégias para conservação é baseado em dados de ocorrência de espécies e as coletas de dados de campo são essenciais (Sousa-Baena et al., 2014; Oliveira et al., 2016), assim como englobar o maior número possível de táxons em estudos de biodiversidade (Oliveira et al., 2017). Porém, mesmo grupos de relevantes serviços ecossistêmicos são sistematicamente negligenciados (Potts et al., 2016; Oliveira et al., 2017). Nesse contexto, o objetivo do presente estudo foi contribuir para o conhecimento de algumas espécies de morcegos e suas moscas ectoparasitas no Parque Nacional da Serra do Pardo, interflúvio Xingu-Tapajós, estado do Pará.

\section{MATERIAL \& MÉTODOS}

\section{ÁREA DE ESTUDO}

O Parque Nacional da Serra do Pardo (PNSP) é uma das UC localizadas na região denominada Terra do Meio, situada nos municípios de Altamira e São Félix do Xingu, à margem do médio rio Xingu, na porção centrooeste do estado do Pará (Figura 1, Tabela 1). Criado em 2005, com 446.552 ha, o PNSP está inserido em uma posição estratégica, a região marginal da Amazônia ainda preservada, de frente à região denominada 'Arco do desmatamento' (ARPA, 2015). Perturbações antrópicas no entorno e, principalmente, nos limites da Área de Proteção Ambiental (APA) Triunfo do Xingu (Doblas, 2015) vêm preocupando a sua manutenção (ARPA, 2015).
A fitofisionomia é representada pela Floresta Tropical Ombrófila Aberta, sub-montanhosa e densa e por Cerrado (Fávaro, 2011). O clima é predominantemente de monção Am (classificação Köppen), com temperatura média em seu regime térmico superior a $22^{\circ} \mathrm{C}$ ao longo de todo o ano, podendo chegar a $40{ }^{\circ} \mathrm{C}$ nos meses de setembro e outubro (ISA, 2003; ARPA, 2015). A elevada pluviosidade (77\% anual em relação à precipitação total) é marcada entre os meses de dezembro a maio, com redução no curto período de junho a novembro no Pará (Moraes et al., 2006).

\section{AMOSTRAGEM DE MORCEGOS E ECTOPARASITAS}

As coletas foram conduzidas no mês de agosto de 2018, durante três noites, uma em cada ponto (P1, P2 e P3; Tabela 1), com utilização de oito redes de neblina $(12 \mathrm{~m} \times 2,5 \mathrm{~m})$. As redes foram abertas ao pôr do sol e mantiveram-se assim durante seis horas, sendo verificadas em intervalos de uma hora (Fleming, 1988). Adicionalmente, foi realizado um dia de busca ativa, com captura manual utilizando puçás (P4; Tabela 1, Figura 2). A busca ativa foi realizada em uma ilha, denominada de Buraco da Arara, localizada no limítrofe da UC, na margem esquerda do rio Xingu. Chegamos ao P4 por meio de informação de moradores locais, que relataram a ocorrência de muitos morcegos nas fendas das rochas. O esforço amostral foi calculado segundo Straube \& Bianconi (2002).

Tabela 1. Pontos amostrados no Parque Nacional da Serra do Pardo, estado do Pará, com a data de coleta, coordenadas geográficas e metodologia empregada.

\begin{tabular}{|c|c|c|c|c|c|}
\hline \multirow[t]{2}{*}{ Ponto } & \multirow{2}{*}{$\begin{array}{c}\text { Data da } \\
\text { amostragem }\end{array}$} & \multicolumn{2}{|c|}{$\begin{array}{l}\text { Coordenadas } \\
\text { (Grau decimal) }\end{array}$} & \multirow{2}{*}{$\begin{array}{l}\text { Método de } \\
\text { amostragem }\end{array}$} & \multirow{2}{*}{ Esforço total } \\
\hline & & Latitude & Longitude & & \\
\hline $\mathrm{P} 1$ & 09/08/2018 & -5.77553 & -52.61872 & Rede de neblina & $1.440 \mathrm{~m}^{2} . \mathrm{h}$ \\
\hline P2 & $10 / 08 / 2018$ & -5.77166 & -52.62146 & Rede de neblina & $1.440 \mathrm{~m}^{2} . \mathrm{h}$ \\
\hline P3 & $13 / 08 / 2018$ & -5.77095 & -52.62637 & Rede de neblina & $1.440 \mathrm{~m}^{2} . \mathrm{h}$ \\
\hline P4 & $12 / 08 / 2018$ & -5.70389 & -52.65472 & Coleta manual & $2 \mathrm{~h}$ \\
\hline
\end{tabular}

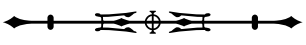




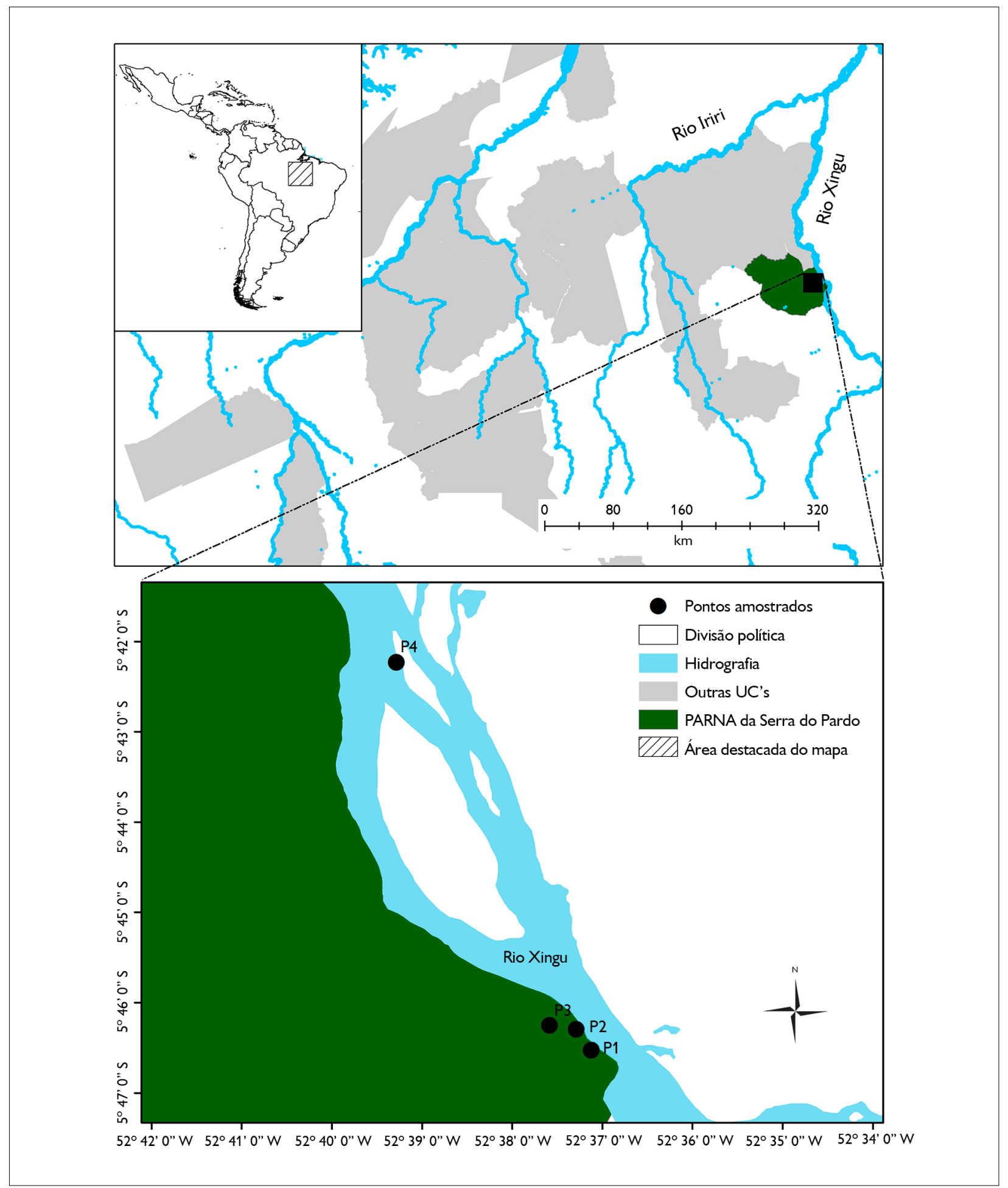

Figura 1. Localização do Parque Nacional Serra do Pardo (PNSP), estado do Pará, Brasil. Pontos amostrados: P1, P2, P3 e P4. Mapa: Thiago Vieira (2019).

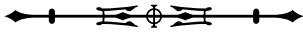



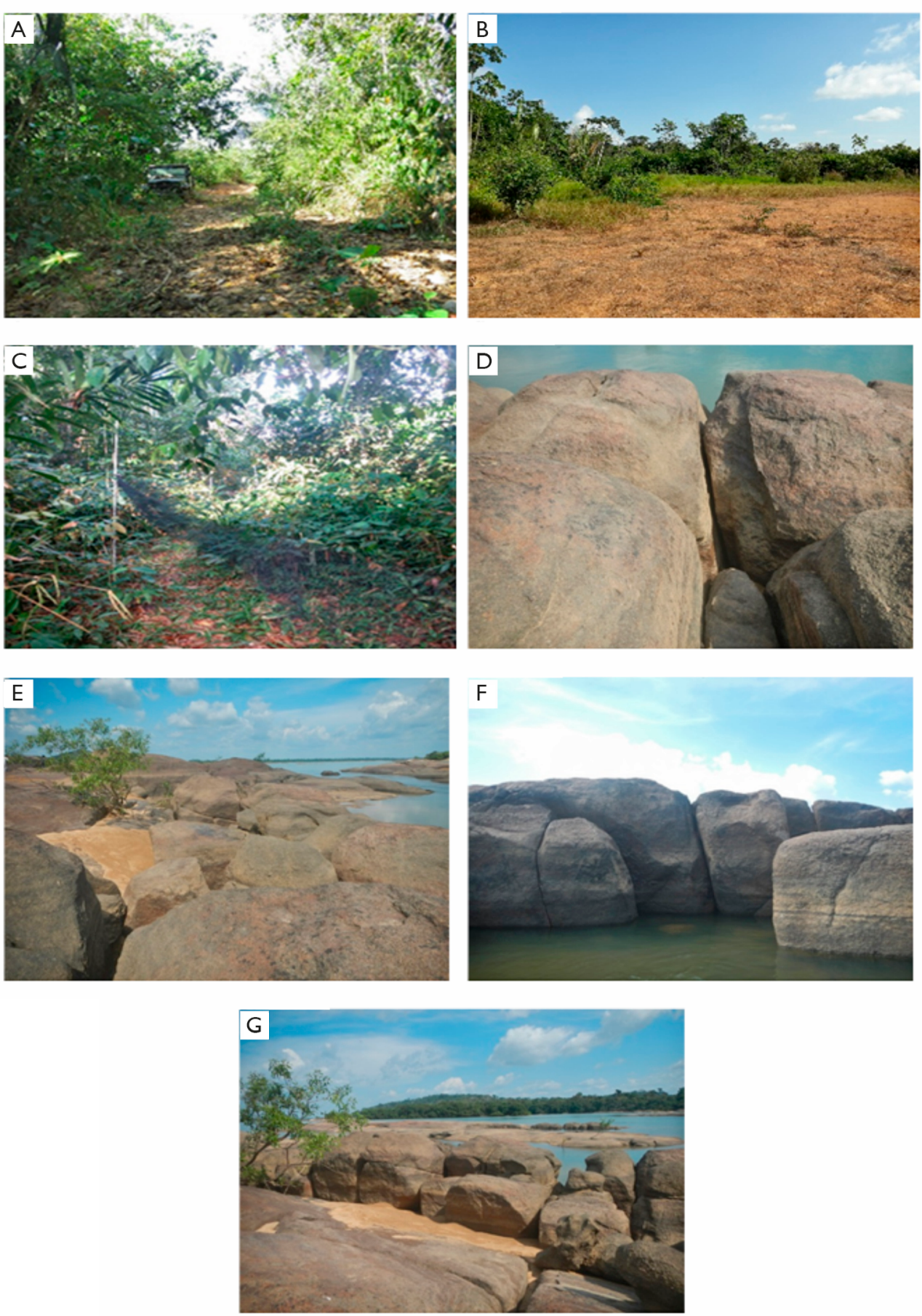

Figura 2. Locais (pontos) amostrados no Parque Nacional Serra do Pardo (PNSP): A) ponto P1; B) ponto P2; C) ponto P3; e ilha do Buraco da Arara (D-G) durante a campanha de agosto de 2018 - ponto P4, com destaque para os aglomerados rochosos em que foram realizadas buscas ativas e captura de morcegos. A ilha do Buraco está localizada no rio Xingu, no limite oriental do Parque Nacional Serra do Pardo, estado do Pará, Brasil. Fotos: acervo ChiroXingu. 
Os morcegos capturados foram acondicionados em sacos de algodão. Após a triagem dos morcegos, os sacos eram revisados, para que as moscas e sementes presentes fossem retiradas, evitando a contaminação das amostras seguintes. Ao menos um casal de cada espécie foi coletado e depositado na Coleção de Morcegos do ChiroXingu (Tabela 2), localizada no Laboratório de Zoologia da Universidade Federal do Pará, campus Altamira, servindo como espécimes-testemunhos, identificadas com a utilização das chaves dicotômicas (e.g., Vizotto \& Taddei, 1973; Gardner, 2008). Cada morcego capturado e não coletado recebeu uma coleira de identificação, numerada com anéis coloridos, conforme Esbérard \& Daemon (1999) e Esbérard et al. (2011). Foi dada preferência para a coleta de um casal de cada espécie, evitando fêmeas grávidas, lactantes e indivíduos jovens. A classificação das guildas alimentares seguiu Kalko et al. (1996), que considera o habitat utilizado pela espécie, o modo de forrageamento e a dieta.

Após a retirada dos morcegos da rede e imediatamente antes da morfometria, foi realizada a busca ativa por ectoparasitos em todo o corpo do indivíduo (pelagem, asas e orelhas). Aqueles encontrados foram extraídos com pinças de ponta fina e pincéis umedecidos em álcool etílico 70\%. Os ectoparasitos foram fixados em álcool etílico 70\% e acondicionados em recipientes individuais, etiquetados de acordo com cada hospedeiro. Em laboratório, os espécimes de moscas foram identificados até o menor nível taxonômico, utilizando bibliografia específica (Guerrero, 1993, 1994, 1995, 1996; Wenzel, 1976) e depositados na Coleção de Dípteras do ChiroXingu (Tabela 3), localizada no Laboratório de Zoologia da Universidade Federal do Pará, campus Altamira. Este estudo foi autorizado pelo Instituto Chico Mendes de Conservação da Biodiversidade (ICMBio) através da licença SEI/ICMBIO - 3661775.

\section{RESULTADOS \& DISCUSSÃO}

Foram amostrados 13 indivíduos de morcegos, 11 com rede de neblina e dois por captura manual (Tabela 2, Figura 3). Com exceção do ponto P4 (onde capturamos os indivíduos durante 2 horas com auxílio de luvas de raspa de couro), os

Tabela 2. Espécies de morcegos capturados no Parque Nacional da Serra do Pardo (PNSP), estado do Pará, Brasil, em 2018. Os morcegos (táxon) estão organizados em família, subfamília e espécie. Além disso, são apresentadas as guildas tróficas e as abundâncias totais e por pontos de coletas (P1, P2, P3 e P4), bem como os números de tombo dos espécimes-testemunhos (TV = Coleção de Morcegos do ChiroXingu, UFPA, campus Altamira).

\begin{tabular}{|c|c|c|c|c|c|c|c|}
\hline Táxon & Guilda trófica & $\mathrm{P} 1$ & $\mathrm{P} 2$ & P3 & P4 & Total & $\begin{array}{l}\text { Espécimes- } \\
\text { testemunhos }\end{array}$ \\
\hline MOLOSSIDAE & Insetívoro aéreo & & & & & & \\
\hline Nyctinomops laticaudatus (É. Geoffroy, 1805) & & - & - & - & 2 & 2 & TV45, TV46 \\
\hline \multicolumn{8}{|l|}{ PHYLLOSTOMIDAE } \\
\hline \multicolumn{8}{|l|}{ Carollinae } \\
\hline Carollia perspicillata (Linnaeus, 1758) & Frugívoro & 2 & 4 & 1 & - & 7 & TV144 \\
\hline Rhinophylla fischerae Carter, 1966 & Frugívoro & - & 1 & - & - & 1 & TV143 \\
\hline \multicolumn{8}{|l|}{ Phyllostominae } \\
\hline Micronycteris sp. & Insetívoro catador & - & 1 & - & - & 1 & - \\
\hline \multicolumn{8}{|l|}{ Stenodermatinae } \\
\hline Artibeus fimbriatus Gray, 1838 & Frugívoro & 1 & - & - & - & 1 & TV141 \\
\hline Platyrrhinus lineatus (É. Geoffroy, 1810) & Frugívoro & - & 1 & - & - & 1 & TV142 \\
\hline Abundância & & 3 & 7 & 1 & 2 & 13 & \\
\hline Riqueza & & 2 & 4 & 1 & 1 & 6 & \\
\hline
\end{tabular}

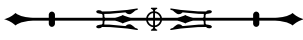


Tabela 3. Espécies de moscas ectoparasitas amostradas nos morcegos capturados no Parque Nacional da Serra do Pardo, estado do Pará, Brasil, em 2018. São apresentadas a riqueza e as abundâncias totais nos quatro pontos de coletas (P1, P2, P3 e P4) e os números de tombo dos espécimes-testemunhos (CX---D = Coleção Dípteras do ChiroXingu, UFPA, campus Altamira).

\begin{tabular}{lcccccc}
\hline \multicolumn{1}{c}{ Táxon } & P1 & P2 & P3 & P4 & Total & Espécimes-testemunhos \\
\hline $\begin{array}{l}\text { STREBLIDAE } \\
\text { Speiseria ambigua Kessel, 1925 }\end{array}$ & - & 1 & - & - & 1 & CX049D \\
$\begin{array}{l}\text { Strebla mirabilis (Waterhouse, 1879) } \\
\text { Trichobius dugesioides dugesioides Wenzel, 1966 }\end{array}$ & 1 & - & 1 & - & 2 & $\begin{array}{c}\text { CX058D } \\
\text { CX036D, CX044D, }\end{array}$ \\
$\begin{array}{l}\text { Abundância } \\
\text { Riqueza }\end{array}$ & 2 & 4 & - & - & 6 & \begin{tabular}{c} 
CX057D, CX090D \\
\hline
\end{tabular} \\
\hline
\end{tabular}

pontos onde foram utilizadas redes de neblina (P1, P2 e P3) tiveram o mesmo esforço amostral: $1.440 \mathrm{~m}^{2}$.h por ponto $\mathrm{e}$ $4.320 \mathrm{~m}^{2}$.h total (Tabela 1). Os resultados não apresentaram espécies inesperadas, e todas as associações entre morcegos e moscas são comuns, conforme discutido nos parágrafos a seguir. Os morcegos capturados representam duas famílias (Phyllostomidae e Molossidae), seis gêneros e seis táxons, com 12 indivíduos identificados em nível de espécie e um em nível de gênero (Tabela 2, Figura 3). O local com maior diversidade foi o ponto 2, com sete indivíduos, distribuídos em quatro táxons (Tabela 2, Figura 3). Somente a espécie Nyctinomops laticaudatus (É. Geoffroy, 1805) teve fêmea capturada e apenas um indivíduo (TV146).

A espécie mais conspícua foi Carollia perspicillata (Linnaeus, 1758), com sete indivíduos amostrados, presente em três dos quatro pontos amostrados (Tabela 2). Não foi possível identificar o espécime de Micronycteris Gray, 1866 em nível de espécie porque o indivíduo fugiu. Durante os trabalhos de campo, um indivíduo adulto e reprodutivamente inativo de C. perspicillata foi coletado com injúrias, possivelmente provenientes de um evento de predação ou briga entre animais da colônia (Figura 3). Quanto à guilda trófica, observamos espécies insetívoras aéreas forrageadoras de espaço aberto e frugívoras, sendo as últimas as mais abundantes (Tabela 2). Para moscas ectoparasitas, registramos a ocorrência apenas da família Streblidae, apresentando três gêneros e três espécies entre os oito indivíduos de moscas coletados (Tabela 3). Todos os ectoparasitas estavam relacionados aos indivíduos de $C$. perspicillata.

O baixo esforço amostral e o reduzido número de capturas impossibilitam testes elaborados para o presente estudo. Contudo, podemos observar uma sugestão do padrão de diversidade observado entre áreas abertas e áreas fechadas. Essa sugestão de padrão é baseada nas observações dos dados brutos. As áreas abertas (P1 e P2) apresentaram maior riqueza de espécies de morcegos, com composição diferente da área fechada (P3). A maior riqueza nas áreas abertas pode ser explicada pelo fato de estas serem ecótonos entre as áreas abertas e as mais densamente florestadas. Ecótonos tendem a apresentar maior riqueza e diversidade de espécies (Whittaker et al., 2001; McCain \& Grytnes, 2010). Além disso, Phyllostomidae é a família de morcegos com maior riqueza na Amazônia (Bernard, 2001; Sampaio et al., 2003; Bernard et al., 2011; Miranda et al., 2015), e suas espécies frugívoras geralmente estão relacionadas à estrutura de vegetação de florestas primárias, sub-bosque e áreas alteradas (Bernard \& Fenton, 2003) e ao comportamento de forrageio (Bernard \& Fenton, 2003; Loayza \& Loiselle, 2008; Bobrowiec \& Gribel, 2009), o que pode facilitar sua captura em redes de neblina (Calouro et al., 2010). Além disso, o método de amostragem utilizado neste estudo é considerado mais eficiente para captura de Phyllostomidae, o que pode ter contribuído para a menor diversidade de guildas (Kunz, 1988; Kalko et al., 1996; Costa et al., 2012).

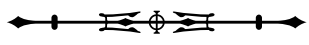



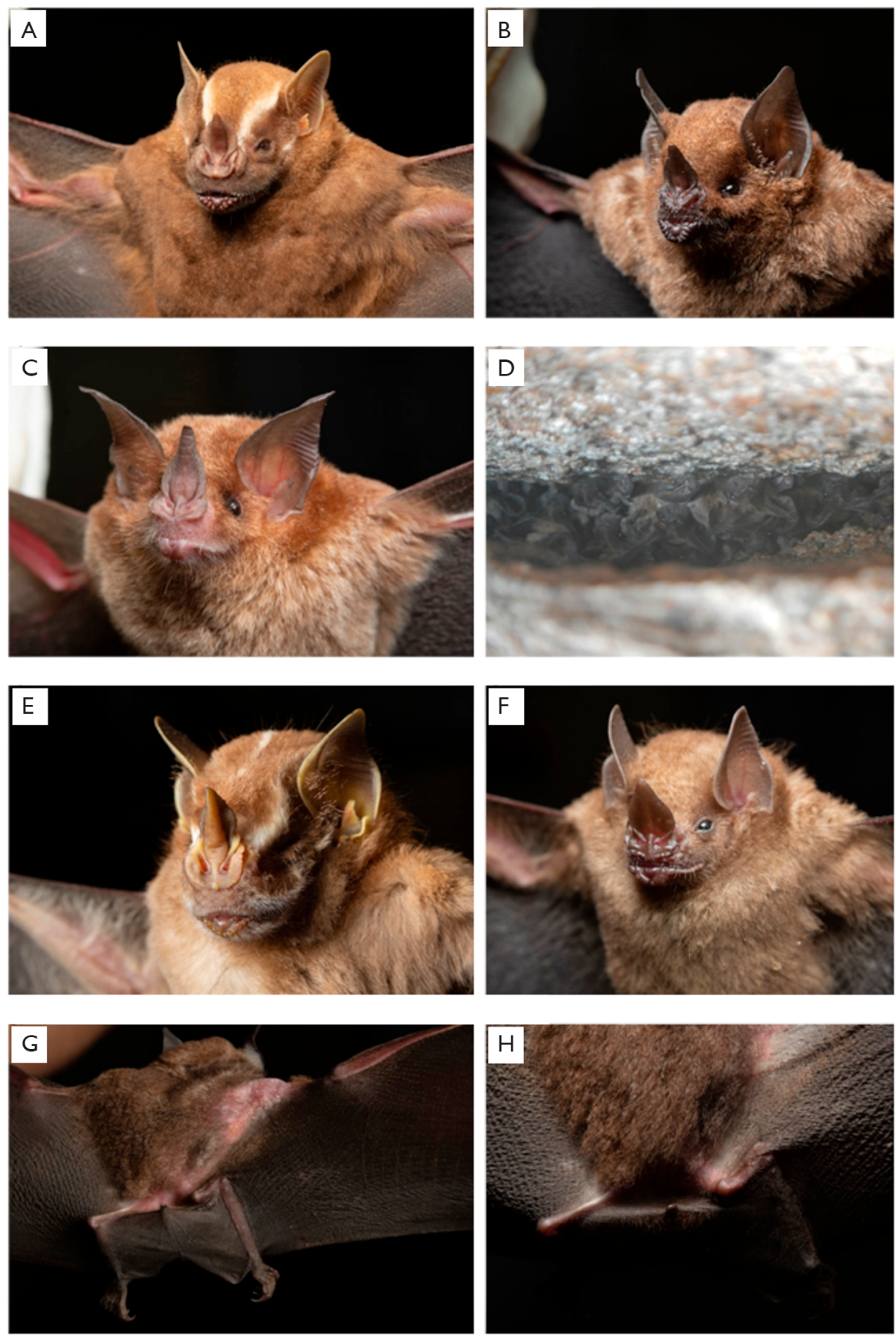

Figura 3. Morcegos capturados no Parque Nacional Serra do Pardo (PNSP): A) Artibeus fimbriatus Gray, 1838; B) Carollia perspicillata (Linnaeus, 1758); C) Micronycteris sp.; D) colônia de Nyctinomops laticaudatus (É. Geoffroy, 1805) observada nas fendas de rochas da ilha Buraco das Araras (P4); E) Platyrrhinus lineatus (É. Geoffroy, 1810); F) Rhinophylla fischerae Carter, 1966; G) detalhe do dorso de um C. perspicillata com marcas de predação; $\mathrm{H}$ ) detalhe da perna direita de um exemplar de $C$. perspicillata com marcas de predação. Fotos: acervo ChiroXingu.

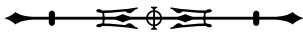


Os dados coletados para esse estudo não são suficientes para indicar a real riqueza de espécies de morcegos que ocorrem no PNSP. No entanto, entre as espécies amostradas, $C$. perspicillata foi a mais abundante. Entre os frugívoros, essa espécie é considerada generalista, com ampla distribuição, sendo abundante na Amazônia (Bernard, 2001; Bernard \& Fenton, 2007; Calouro et al., 2010; Miranda et al., 2015; Verde et al., 2018). As associações observadas aqui entre $C$. perspicillata com Speiseria ambigua Kessel, 1925, Strebla mirabilis (Waterhouse, 1879) e Trichobius dugesioides Wenzel, 1966 já foram previamente descritas, tanto para outros biomas como para a Amazônia (e.g., Graciolli \& Aguiar, 2002; Dias et al., 2009; Santos, C. et al. , 2009; Urbieta et al., 2018; Torres et al., 2019). Ambientes de baixa qualidade e a disponibilidade de recursos podem reduzir a fidelidade de C. perspicillata ao abrigo, provocando maior contato com outras espécies de morcegos, o que poderia influenciar no compartilhamento de moscas ectoparasitas (Bush \& Lotz, 2000; Santos, F. et al., 2012; Barbier \& Graciolli, 2016; Urbieta et al., 2018). Considerando a escassez de estudos sobre ectoparasitas em morcegos da Amazônia (e.g., Santos, F. et al., 2012; Lourenço et al., 2016; Urbieta et al., 2018), pesquisas de longo prazo, com mais de um método de amostragem e ampliação do esforço e de pontos amostrais, são fundamentais para aumentar o conhecimento da diversidade de morcegos e a inter-relação com seus ectoparasitos. Além disso, estudos de longo prazo permitiriam o acompanhamento da qualidade ambiental na área protegida.

\section{AGRADECIMENTOS}

$O$ presente trabalho foi realizado com apoio da Coordenação de Aperfeiçoamento de Pessoal de Nível Superior - Brasil (CAPES) - código de financiamento 001. Dois revisores anônimos contribuíram com importantes sugestões e comentários que melhoraram significativamente a qualidade do presente estudo. Agradecemos também ao Programa de Pós-Graduação em Biodiversidade e Conservação (PPGBC), que contribuiu financeiramente na coleta dos dados, ao Instituto Chico Mendes de Conservação da Biodiversidade (ICMBio) e ao Instituto Brasileiro do Meio Ambiente e dos Recursos Naturais Renováveis (IBAMA), pelo o apoio na pesquisa.

\section{REFERÊNCIAS}

ALEIXO, A., 2009. Lacunas de conhecimento, prioridades de pesquisa e perspectivas futuras na conservação de aves na Amazônia brasileira. In: A. DE LUCA, P. F. DEVELEY, G. A. BENCKE \& J. M. GOERCK (Ed.): Áreas importantes para a conservação das aves no Brasil: parte II - Amazônia, Cerrado e Pantanal: 39-49. SAVE Brasil, São Paulo.

BARBIER, E. \& G. GRACIOLLI, 2016. Community of bat flies (Streblidae and Nycteribiidae) on bats in the Cerrado of CentralWest Brazil: hosts, aggregation, prevalence, infestation intensity, and infracommunities. Studies on Neotropical Fauna and Environment 51(3): 176-187. DOI: https://doi.org/10.1080/016 50521.2016.1215042

BARROS, L. P., 2010. A história do Parque Nacional Serra do Pardo. WWF-Brasil. Disponível em: https://www.wwf.org.br/ natureza_brasileira/areas_prioritarias/amazonia1/nossas solucoes na_amażonia/exp/2010__expedicao_cientifica_terra_do_ mēio/?27226/A-historia-do--Parque-Nacional-Serra-do-Pardo. Acesso em: 22 novembro 2019.

BERNARD, E., 2001. Vertical stratification of bat communities in primary forests of Central Amazon, Brazil. Journal of Tropical Ecology 17(1): 115-126. DOI: https://doi.org/10.1017/ S0266467401001079

BERNARD, E. \& M. B. FENTON, 2003. Bat mobility and roosts in a fragmented landscape in Central, Amazonia, Brazil. Biotropical 35(2): 262-277. DOI: https://doi.org/10.1111/j.1744-7429.2003. tb00285.x

BERNARD, E. \& M. B. FENTON, 2007. Bats in a fragmented landscape: species composition, diversity and habitat interactions in savannas of Santarém, Central Amazonia, Brazil. Biological Conservation 134(3): 332-343. DOI: https://doi.org/10.1016/j. biocon.2006.07.021

BERNARD, E., V. C. TAVARES \& E. SAMPAIO, 2011. Compilação atualizada das espécies de morcegos (Chiroptera) para a Amazônia brasileira. Biota Neotropica 11(1): 35-46. DOI: http://dx.doi. org/10.1590/S1676-06032011000100003

BOBROWIEC, P. E. D. \& R. GRIBEL, 2009. Effects of different secondary vegetation types on bat community composition in Central Amazonia, Brazil. Animal Conservation 13(2): 204-216. DOI: https://doi.org/10.1111/J.1469-1795.2009.00322.x 
BRASIL, 2005. Decreto de 17 de fevereiro de 2005. Cria o Parque Nacional da Serra do Pardo, localizado nos Municípios de Altamira e São Félix do Xingu, Estado do Pará, e dá outras providências. Diário Oficial da União. Disponível em: http://www.planalto.gov. br/ccivil_03/_Ato2004-2006/2005/Dnn/Dnn10451.htm. Acesso em: 16 novembro 2020.

BUSH, A. O. \& J. M. LOTZ, 2000. The ecology of "crowding". Journal of Parasitology 86(2): 212-213. DOI: https://doi. org/10.1645/0022-3395(2000)086[0212:TEOC]2.0.CO;2

CALOURO, A. M., F. G. A. SANTOS, C. L. FAUSTINO, S. F. SOUZA, B. M. LAGUE, R. M. T. SILVA, G. J. L. SANTOS \& A. O. CUNHA, 2010. Riqueza e abundância de morcegos capturados na borda e no interior de um fragmento florestal do estado do Acre, Brasil. Biotemas 4(23): 109-117. DOI: https://doi. org/10.5007/2175-7925.2010v23n4p109

CLEVELAND, C. J., M. BETKE, P. FEDERICO, J. D. FRANK, T. G. HALLAM, J. HORN, J. D. LÓPEZ JR., G. F. MCCRACKEN, R. A. MEDELLÍN, A. MORENO-VALDEZ, C. G. SANSONE, J. K. WESTBROOK \& T. H. KUNZ, 2006. Economic value of the pest control service provided by Brazilian free-tailed bats in south-central Texas. Frontiers in Ecology and the Environment 4(5): 238-243. DOI: https://doi.org/10.1890/1540-9295(2006)004[0238:EVOTP C]2.0.CO;2

COLLEN, B., M. RAM, T. ZAMIN \& L. MCRAE, 2008. The tropical biodiversity data gap: addressing disparity in global monitoring. Tropical Conservation Science 1(2): 75-88. DOI: https://doi. org/10.1177/194008290800100202

COSTA, L. M., J. L. LUZ \& C. E. L. ESBÉRARD, 2012. Riqueza de morcegos insetívoros em lagoas no Estado do Rio de Janeiro, Brasil. Papéis Avulsos de Zoologia 52(2): 7-19. DOI: https://doi. org/10.1590/S0031-10492012000200001

DEVELEY, P. F., 2009. Áreas importantes para a conservação das aves no Brasil. In: C. DEVENISH, D. F. DÍAZ FERNÁNDEZ, R. P. CLAY, I. DAVIDSON \& Y. I. ZABALA (Ed.): Áreas importantes para a conservação das aves no Brasil: parte II: 1-8. SAVE Brasil, São Paulo.

DIAS, P. A., C. L. C. SANTOS, F. S. RODRIGUES, L. C. ROSA, K. S. LOBATO \& J. M. M. REBELO, 2009. Espécies de moscas ectoparasitas (Diptera, Hippoboscoidea) de morcegos (Mammalia, Chiroptera) no estado do Maranhão. Revista Brasileira de Entomologia 53(1): 128-133. DOI: http://dx.doi.org/10.1590/S008556262009000100027

DOBLAS, J., 2015. Rotas do saque: violações e ameaças à integridade territorial da Terra do Meio (PA): 1-48. Instituto Socioambiental, São Paulo.

ESBÉRARD, C. E. L. \& C. DAEMON, 1999. Um novo método para marcação de morcegos. Chiroptera Neotropical 5(1-2): 116-117.
ESBÉRARD, C. E. L., I. P. LIMA, P. H. NOBRE, S. L. ALTHOFF, T. JORDÃO-NOGUEIRA, D. DIAS, F. CARVALHO, M. E. FABIÁN, M. L. SEKIAMA \& A. S. SOBRINHO, 2011. Evidence of vertical migration in the Ipanema bat Pygoderma bilabiatum (Chiroptera: Phyllostomidae: Stenodermatinae). Zoologia 28(6): 717-724. DOI: http://dx.doi.org/10.1590/S1984-46702011000600004

ESCADA, M. I. S., I. C. G. VIEIRA, S. A. KAMPEL, R. ARAÚJO, J. B. VEIGA, A. P. D. AGUIAR, I. VEIGA, M. OLIVEIRA, J. L. G. PEREIRA, A. CARNEIRO FILHO, P. M. FEARNSIDE, A. VENTURIERI, F. CARRIELLO, M. THALES, T. S. G. CARNEIRO, A. M. V. MONTEIRO \& G. CÂMARA, 2005. Processos de ocupação nas novas fronteiras da Amazônia: o interflúvio do Xingu/Iriri. Estudos Avançados 19(54): 9-23. DOI: http://dx.doi.org/10.1590/S010340142005000200002

FÁVARO, F. D. L., 2011. Aves do Parque Nacional da Serra do Pardo, Pará, Brasil: levantamento inicial. Ornithologia 4(2): 91-103.

FLEMING, T. H., 1988. The short-tailed fruit bat: a study in plantanimal interactions: 1-365. University of Chicago Press, Chicago.

FLEMING, T. H. \& E. R. HEITHAUS, 1981. Frugivorous bats, seed shadows, and the structure of tropical forests. Biotropica 13(2): 45-53. DOI: https://doi.org/10.2307/2388069

FRANK, R., J. MÜNSTER, J. SCHULZE, A. LISTON \& S. KLIMPEL, 2014. Macroparasites of Microchiroptera: bat ectoparasites of Central and South America. In: S. KLIMPEL \& H. MEHLHORN (Ed.): Bats (Chiroptera) as vectors of diseases and parasites: 87-130. Springer (Parasitology Research Monographs, vol. 5), Berlin, Heidelberg. DOI: https://doi.org/10.1007/978-3-642-39333-4_5

GARBINO, G. S. T., R. GREGORIN, I. P. LIMA, L. LOUREIRO, L. M. MORAS, R. MORATELLI, M. R. NOGUEIRA, A. C. PAVAN, V. C. TAVARES \& A. L. PERACCHI, 2020. Updated checklist of Brazilian bats: versão 2020. Comitê da Lista de Morcegos do Brasil/Sociedade Brasileira para o Estudo de Quirópteros. Disponível em: https:// www.sbeq.net/lista-de-especies. Acesso em: 16 novembro 2020.

GARDNER, A. L. (Ed.), 2008. Mammals of South America: vol. 2: marsupials, xenarthrans, shrews, and bats: 1-690. University of Chicago Press, Chicago and Londres.

GHANEM, S. J. \& C. C. VOIGT, 2012. Increasing awareness of ecosystem services provided by bats. Advances in the Study of Behavior 44(7): 279-302. DOI: https://doi.org/10.1016/B978-012-394288-3.00007-1

GRACIOLLI, G., 2020. Streblidae. In: Catálogo Taxonômico da Fauna do Brasil. PNUD. Disponível em: http://fauna.jbrj.gov.br/ fauna/faunadobrasil/2624. Acesso em: 23 março 2020.

GRACIOLLI, G. \& L. S. AGUIAR, 2002. Ocorrência de moscas ectoparasitas (Diptera, Streblidae e Nycteribiidae) de morcegos (Mammalia, Chiroptera) no cerrado de Brasília, Distrito Federal, Brasil. Revista Brasileira de Zoologia 19(1): 177-181. DOI: http:// dx.doi.org/10.1590/S0101-81752002000500012

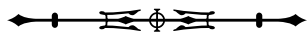


GRACIOLLI, G. \& G. V. BIANCONI, 2007. Moscas ectoparasitas (Diptera, Streblidae e Nycteribiidae) em morcegos (Mammalia, Chiroptera) em área de floresta com Araucária no estado do Paraná, sul do Brasil. Revista Brasileira de Zoologia 24(1): 246-249. DOI: http://dx.doi.org/10.1590/S0101-81752007000100033

GRACIOLLI, G., A. G. AUTINO \& G. L. CLAPS, 2007. Catalogue of American Nycteribiidae (Diptera, Hippoboscoidea). Revista Brasileira de Entomologia 51(2): 142-159. DOI: http://dx.doi. org/10.1590/S0085-56262007000200004

GUERRERO, R., 1993. Catalogo de los Streblidae (Diptera: Pupipara) parasitos de murcielago (Mammalia: Chiroptera) del Nuevo mundo. I. Clave para los generos y Nycterophilinae. Acta Biologica Venezolana 14(4): 61-75.

GUERRERO, R., 1994. Catalogo de los Streblidae (Diptera: Pupipara) parasitos de murcielagos (Mammalia: Chiroptera) del Nuevo mundo. IV. Trichobiinae con alas desarrolladas. Boletín de Entomología Venezolana 9(2): 161-192.

GUERRERO, R., 1995. Catalogo de los Streblidae (Diptera: Pupipara) parasitos de murcielagos (Mammalia: Chiroptera) del Nuevo mundo. III. Los grupos: Dugessi, Dunni y Phyllostomae del genero Trichobius Gervais, 1844. Acta Biologica Venezolana 15(3-4): 1-27.

GUERRERO, R., 1996. Catalogo de los Streblidae (Diptera: Pupipara) parasitos de murcielagos (Mammalia: Chiroptera) del Nuevo mundo. VI. Streblinae. Acta Biologica Venezolana 16(2): 1-25.

HOPKINS, M. J. G., 2007. Modelling the known and unknown plant biodiversity of the Amazon Basin. Journal of Biogeography 34: 1400-1411. DOI: https://doi.org/10.1111/j.1365-2699.2007.01737.x

INSTITUTO CHICO MENDES DE CONSERVAÇÃO DA BIODIVERSIDADE (ICMBio), 2012. Plano de manejo participativo Reserva Extrativista Rio Xingu: 1-160. ICMBio/MMA, Altamira. Disponível em: https://www.icmbio.gov.br/portal/images/stories/ imgs-unidades-coservacao/PM-RESEX-Rio-Xingu-2012.pdf. Acesso em: 16 novembro 2020.

INSTITUTO SOCIOAMBIENTAL (ISA), 2003. Projeto Realização de estudos preliminares e formulação de uma proposta técnica para a implantação de um mosaico de Unidades de Conservação no Médio Xingu. Relatório final: 1-129. Disponível em: https://www. socioambiental.org/sites/blog.socioambiental.org/files/relatorios/ rel2003.pdf. Acesso em: 16 novembro 2020.

KALKA, M. B., A. R. SMITH \& E. K. V. KALKO, 2008. Bats limit arthropods and herbivory in a tropical forest. Science 320(5872): 71. DOI: https://doi.org/10.1126/science.1153352

KALKO, E. K. V., C. O. HANDLEY JR. \& D. HANDLEY, 1996. Organization, diversity and long-term dynamics of a neotropical bat community. In: M. L. CODY \& J. A. SMALLWOOD (Ed.): Longterm studies of vertebrate communities: 503-553. Academic Press, New York.
KIER, G., J. MUTKE, E. DINERSTEIN, T. H. RICKETTS, W. KÜPER, H. KREFT \& W. BARTHLOTT, 2005. Global patterns of plant diversity and floristic knowledge. Journal of Biogeography 32(7): 1107-1116. DOI: https://doi.org/10.1111/ j.1365-2699.2005.01272.x

KUNZ, T. H., 1988. Methods of assessing the availability of prey to insectivorous bats. In: T. H. KUNZ (Ed.): Ecological and behavioral methods for the study of bats: 191-210. Smithsonian Institution Press, Washington.

KUNZ, T. H., E. B. TORREZ, D. BAUER, T. LOBOVA \& T. H. FLEMING, 2011. Ecosystem services provided by bats. Annals of the New York Academy of Sciences 1223(1): 1-38. DOI: https:// doi.org/10.1111/j.1749-6632.2011.06004.x

LOAYZA, A. P. \& B. A. LOISELLE, 2008. Preliminary information on the home range and movement patterns of Sturnira lilium (Phyllostomidae) in a naturally fragmented landscape in Bolivia. Biotropica 40(5): 630-635. DOI: https://doi.org/10.1111/j.17447429.2008.00422.x

LOURENÇO, E. C., J. C. ALMEIDA, K. M. FAMADAS, 2016. Richness of ectoparasitic flies (Diptera: Streblidae) of bats (Chiroptera) - a systematic review and meta-analysis of studies in Brazil. Parasitology Research 115: 4379. DOI: https://doi. org/10.1007/s00436-016-5223-y

MCCAIN, C. M. \& J. A. GRYTNES, 2010. Elevational gradients in species richness. In: Encyclopedia of life sciences: 1-10. John Wiley \& Sons, Chichester, UK. DOI: http://dx.doi. org/10.1002/9780470015902.a0022548

MIRANDA, J. M. D., L. ZAGO, F. CARVALHO, M. B. G. RUBIO \& I. P. BERNARDI, 2015. Morcegos (Mammalia: Chiroptera) da região do Médio Rio Teles Pires, Sul da Amazônia, Brasil. Acta Amazônica 45(1): 89-100. DOI: http://dx.doi.org/10.1590/18094392201400583

MORAES, B. C., J. M. N. COSTA, A. C. L. COSTA \& M. H. COSTA, 2006. Variação espacial e temporal da precipitação no estado do Pará. Acta Amazonica 35(2): 207-214. DOI: http:// dx.doi.org/10.1590/S0044-59672005000200010

NOGUEIRA, M. R., I. P. LIMA, R. MORATELLI, V. C. TAVARES, R. GREGORIN \& A. L. PERACCHI, 2014. Checklist of Brazilian bats, with comments on original records. Check List 10(4): 808-821. DOI: http://dx.doi.org/10.15560/10.4.808

OliVEIRA, U., A. P. PAGLIA, A. D. BRESCOVIT, C. J. B. CARVALHO, D. P. SILVA, D. T. REZENDE, F. S. F. LEITE, J. A. N. BATISTA, J. P. P. P. BARBOSA, J. R. STEHMANN, J. S. ASCHER, M. F. VASCONCELOS, P. DE MARCO, P. LÖWENBERG-NETO, P. G. DIAS, V. G. FERRO \& A. J. SANTOS, 2016. The strong influence of collection bias on biodiversity knowledge shortfalls of Brazilian terrestrial biodiversity. Diversity and Distributiions 22(12): 1232 1244. DOI: https://doi.org/10.1111/ddi.12489

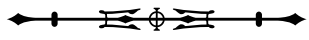


OLIVEIRA, U., B. S. SOARES-FILHO, A. P. PAGLIA, A. D. BRESCOVIT, C. J. B. CARVALHO, D. P. SILVA, D. T. REZENDE, F. S. F. LEITE, J. A. N. BATISTA, J. P. P. P. BARBOSA, J. R. STEHMANN, J. S. ASCHER, M. F. VASCONCELOS, P. DE MARCO, P. LÖWENBERG-NETO, V. G. FERRO \& A. J. SANTOS, 2017. Biodiversity conservation gaps in the Brazilian protected areas. Scientific Reports 7(1): 1-9. DOI: https://doi.org/10.1038/s41598017-08707-2

PAGLIA, A. P., G. A. B. FONSECA, A. B. RYLANDS, G. HERRMANN, L. M. S. AGUIAR, A. G. CHIARELLO, Y. L. R. LEITE, L. P. COSTA, S. SICILIANO, M. C. M. KIERULFF, S. L. MENDES, V. C. TAVARES, R. A. MITTERMEIER \& J. L. PATTON, 2012. Lista anotada dos mamíferos do Brasil/Annotated checklist of Brazilian mammals: 2. ed.: 1-76. Conservation International, Arlington.

POTTS, S. G., V. IMPERATRIZ-FONSECA, H. T. NGO, M. A AIZEN, J. C. BIESMEIJER, T. D. BREEZE, L. V. DICKS, L. A. GARIBALDI, R. HILL, J. SETTELE \& A. J. VANBERGEN, 2016. Safeguarding pollinators and their values to human well-being. Nature 540(7632): 220-229. DOI: https://doi.org/10.1038/ nature20588

PREVEDELLO, J. A., G. GRACIOLLI \& C. J. B. CARVALHO, 2005. A fauna de dípteros (Streblidae e Nycteribiidae) ectoparasitas de morcegos (Chiroptera) do estado do Paraná, Brasil: composição, distribuição e áreas prioritárias para novos estudos. Biociências 13: 193-209.

PROGRAMA ÁREAS PROTEGIDAS DA AMAZÔNIA (ARPA), 2015. Plano de manejo do Parque Nacional da Serra do Pardo: 1-226. ICMBio/MMA, Brasília. Disponível em: https://www.icmbio. gov.br/portal/images/stories/DCOM_plano_de_manejo_Parna Serra_do_Pardo_18092015.pdf. Acesso em: 19 novembro 2019.

RUI, A. M. \& G. GRACIOLLI, 2005. Moscas ectoparasitas (Diptera, Streblidae) de morcegos (Chiroptera, Phyllostomidae) no sul do Brasil: associações hospedeiros-parasitos e taxas de infestação. Revista Brasileira de Zoologia 22(2): 438-444. DOI: http://dx.doi. org/10.1590/S0101-81752005000200021

SAMPAIO, E. M., E. K. V. KALKO, E. BERNARD, B. RODRÍGUEZHERRERA \& C. O. HANDLEY, 2003. A biodiversity assessment of bats (Chiroptera) in a tropical lowland rainforest of Central Amazonia, including methodological and conservation considerations. Studies on Neotropical Fauna and Environment 38(1): 17-31. DOI: http:// dx.doi.org/10.1076/snfe.38.1.17.14035

SANTOS, C. L. C., P. A. DIAS, F. S. RODRIGUES, K. LOBATO, L. C. ROSA, T. G. OLIVEIRA \& J. M. M. REBÊLO, 2009. Moscas ectoparasitas (Diptera: Streblidae) de morcegos (Mammalia: Chiroptera) do município de São Luís, MA: taxas de infestação e associações parasito-hospedeiro. Neotropical Entomology 38(5): 595-601. DOI: http://dx.doi.org/10.1590/ S1519-566X2009000500006
SANTOS, F. G. A., A. M. CALOURO, S. F. SOUZA, B. M. LAGUE, R. MARCIENTE, R. C. L. FAUSTINO, G. J. L. SANTOS \& A. O. CUNHA, 2012. Ectoparasitismo em uma assembleia de morcegos em um fragmento florestal no estado do Acre, Brasil. Acta Veterinaria Brasilica 6(3): 211-218.

SILVA, J. R. R. \& H. ORTÊNCIO FILHO, 2011. Diversity of ectoparasitic dipterans (Insecta, Diptera) in bats (Chiroptera, Mammalia) from the Perobas Biological Reserve in Paraná, south Brazil. Iheringia. Série Zoologia 101(3): 220-224. DOI: https://doi. org/10.1590/S0073-47212011000200009

SIMMONS, N. B. \& A. L. CIRRANELLO, 2020. Bat species of the world: a taxonomic and geographic database. Disponível em: https:// www.batnames.org/. Acesso em: 16 novembro 2020.

SOUSA-BAENA, M. S., L. C. GARCIA \& A. T. PETERSON, 2014. Completeness of digital accessible knowledge of the plants of Brazil and priorities for survey and inventory. Diversity and Distributions 20(4): 369-381. DOI: https://doi.org/10.1111/ddi.12136

STRAUBE, F. C. \& G. V. BIANCONI, 2002. Sobre a grandeza e a unidade utilizada para estimar esforço de captura com utilização de redes-de-neblina. Chiroptera Neotropical 8(1-2): 150-152.

TORRES, J. M., G. L. URBIETA, L. B. M. ALMEIDA, D. K. F. SOARES \& E. A. C. ANJOS, 2019. Moscas ectoparasitas (Diptera, Streblidae) de morcegos (Mammalia, Chiroptera) em um remanescente periurbano de Cerrado: composição da comunidade, prevalência, intensidade de infestação e especificidade. Iheringia. Série Zoologia 109: e2019006. DOI: https://doi.org/10.1590/1678-4766e2019006

URBIETA, G. L., J. M. TORRES, E. A. C. ANJOS, C. M. E. CARVALHO \& G. GRACIOLLI, 2018. Parasitism of bat flies (Nycteribiidae and Streblidae) on bat in urban environments: lower prevalence, infracommunities, and specificity. Acta Chiropterologica 20(2): 511-518. DOI: https://doi.org/10.3161/15081109ACC2018.20.2.021

VELÁSQUEZ, C., A. V. BOAS \& S. SCHWARTZMAN, 2006. Desafio para a gestão ambiental integrada em território de fronteira agrícola no oeste do Pará. Revista de Administração Pública 40(6): 10611075. DOI: http://dx.doi.org/10.1590/S0034-76122006000600007

VERDE, R. S., R. C. SILVA \& A. M. CALOURO, 2018. Activity patterns of frugivorous phyllostomid bats in an urban fragment in southwest Amazonia, Brazil. Ilheringia. Série Zoologia 108: 1-7. DOI: http://dx.doi.org/10.1590/1678-4766e2018016

VIZOTTO, L. D. \& V. A. TADDEI, 1973. Chave para determinação de quirópteros brasileiros. Boletim de Ciências, Faculdade de Filosofia, Ciências e Letras 1: 1-72.

WENZEL, R. L., 1976. The streblid batflies of Venezuela (Diptera: Streblidae). Brigham Young University Science Bulletin, Biological Series 20(4): 1. Disponível em: https://scholarsarchive.byu.edu/ byuscib/vol20/iss4/1. Acesso em: 16 novembro 2020.

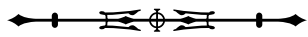


WHITTAKER, R. J., K. J. WILLIS \& R. FIELD, 2001. Scale and species richness: towards a general, hierarchical theory of species diversity. Journal of Biogeography 28(4): 453-470. DOI: https:// doi.org/10.1046/j.1365-2699.2001.00563.x

WILLIAMS-GUILLÉN, K., I. PERFECTO \&J. VANDERMEER, 2008. Bats limit insects in a Neotropical agroforestry system. Science 320(5872): 70. DOI: https://doi.org/10.1126/science.1152944
$X U$, W., Y. XIAO, J. ZHANG, W. YANG, LU. ZHANG, V. HULL, Z. WANG, H. ZHENG, J. LIU, S. POLASKY, L. JIANG, Y. XIAO, X. SHI, E. RAO, F. LU, X. WANGA, G. C. DAILY \& Z. OUYANG, 2017. Strengthening protected areas for biodiversity and ecosystem services in China. Proccedings of the National Academy of Sciences of the United States of America 114: 1-6. DOI: https://doi.org/10.1073/ pnas. 1620503114 
